\title{
Cancer Genome Explosion and Systems Biology: Impact on Surgical Oncology?
}

\author{
Dimitrios H. Roukos, MD, PhD ${ }^{1,2}$ \\ ${ }^{1}$ Personalized Cancer Medicine, Biobank, Ioannina University, Ioannina, Greece; ${ }^{2}$ Department of Surgery, \\ Ioannina University School of Medicine, Ioannina, Greece
}

Each human being and each cancer patient is unique. Based on this assessment provided by the current personal genomics revolution, the emerging conceptual design is great. By identifying the genetics, genomics, and epigenomics variants underlying cancer phenotype, we would move forward toward improving surgical oncology. A decade after the first draft of human full-genome sequence, what is the progress of "big" biology and science? How do these advances influence surgical oncology and what are the challenges to overcome in order to achieve a dramatic improvement in the personalized prevention and treatment of solid cancer?

\section{HUMAN AND CANCER GENOME REVOLUTION}

As we are at the end of the first post-genome decade, we are now facing an explosion in DNA sequencing technology. As the costs dramatically drop, and the sequencing data quality is improved, two dozen complete sequence human genomes have been published and close to 200 unpublished ones are finished. ${ }^{1}$ At least three fully sequenced cancer genomes, including breast, lung, and melanoma cancer, have been published. ${ }^{2}$ All these data now consistently change our understanding of genetic background and molecular mechanisms underlying malignant disease. Cancer is much more complex and heterogeneous than we have supposed. ${ }^{2,3}$ A huge number of mutations are involved in tumorigenesis and metastasis, and it is likely that in most cases complex gene-gene, gene-environment, and intratumoral cell-cell interactions drive the oncological outcome rather than a simple linear

(C) Society of Surgical Oncology 2010

Published Online: 2 October 2010

D. H. Roukos, MD, PhD

e-mail: droukos@uoi.gr genotype-phenotype relationship. ${ }^{2-5}$ Although Collins and Venter agree that this genomic revolution has not yet changed medical practice, it is important to note that the new genomics-based current knowledge now shapes much more rational innovation strategies to successfully prevent and treat cancer in the future. 6,7

\section{STANDARDS IN SURGICAL ONCOLOGY}

Based on advances in cancer biology and molecular oncology and comparative-effectiveness research (CER) using randomized controlled trials (RCTs) and meta-analyses as tools for objective assessments, many prevention and treatment strategies for the most common cancers have been standardized over the last years.

\section{Risk Prediction and Prevention}

Screening programs for the general population, including mammography for breast cancer, colonoscopy for colorectal cancer, and prostate-specific antigen (PSA) levels for prostate cancer have been standardized. This has substantially increased the rates of early-stage cancer diagnosis and saved the lives of thousands of patients. However, many low-risk individuals undergo an unnecessary screening, and often false-positive results lead to nonuseful biopsies. For example, even for the most popular and widely used PSA screening, updated evidence by the U.S. Preventive Services Task Force shows that PSA screening is associated with psychological harms, and its potential benefits remain uncertain. ${ }^{8}$ The current lack of robust biomarkers in cancer is not surprising given the emerging evidence for the complexity of tumorigenesis. ${ }^{2-7}$

Despite these limitations, prophylactic surgery has recently gained considerable attention for the prevention of hereditary breast-ovarian cancer, colorectal cancer (Lynch 
syndrome), and diffuse gastric cancer for individuals who test positive for heritable mutations in BRCA1/BRCA2, mismatch-repair genes, and $C D H 1$ gene, respectively. However, despite its efficacy, prophylactic surgery has limitations including negative effect on quality of life; roughly $25 \%$ of mutation carriers never develop cancer in their life and thus experience an unnecessary surgery. In addition, these hereditary syndromes are rare and the vast majority of individuals who will develop cancer cannot be predicted. ${ }^{9}$

\section{Treatment}

In cancer treatment, tumor-node-metastasis (TNM) staging system and clinicopathologic features have been the standard tools for decisions made on surgery, radiotherapy, and systemic chemotherapy. Based on evidence from CER, the extent of surgery for complete tumor resection (R0) of the primary tumors and regional lymph nodes have been standardized. An R0 resection minimizes local and nodal residual disease and alone or with adjuvant radiotherapy reduces locoregional recurrence and improves survival. Adjuvant chemotherapy, usually in tumor stages II and III, further decreases locoregional and distant recurrence through elimination of disseminated cancer cells and micrometastatic disease at distant sites. Yet, the alarmingly high recurrence rates with poor cure rates, particularly in stage III after modern multimodal treatment of major cancers such as lung, breast, and colorectal cancer drive current translational research for developing new more effective therapies.

\section{TARGETED DRUGS}

Cell proliferation, growth, survival, and apoptosis (death) are regulated by signals that are transmitted from cell surface receptors to its nucleus through various signalling pathways. When mutations occur and accelerate key gene components of these downstream pathways, they deregulate the signal transduction and the above cellular processes including angiogenesis, causing cancer development. The concept of the inhibition of key signalling pathways by biologically targeted agents has rapidly evolved through advances in biotechnology and the pharmaceutical industry over the last decade.

The potential ability of druggable targets to block the function of only cancer cells and not healthy cells has created an overenthusiasm with a rapidly growing list of approved drugs and many others undergoing clinical evaluation. The present generation of drugs includes two major categories: monoclonal antibodies and small molecule tyrosine kinase inhibitors (TKIs). Among the most popular antibodies are the trastuzumab for breast cancer and gastric cancer. ${ }^{9}$ The anti-EGFR cetuximab and panitumumab for colorectal cancer and the TKIs erlotinib or gefitinib for non-small-cell lung cancer (NSCLC).

\section{Efficacy and Limitations}

Table 1 summarizes the most commonly used targeted drugs. Recent phase III RCTs have demonstrated some efficacy in advanced or metastatic setting in selected patients measured as progression-free survival (PFS) without any overall survival (OS) benefit. For example, cetuximab or panitumumab added to chemotherapy has improved PFS in KRAS wild-type only metastatic colorectal cancer and gefitinib added to chemotherapy in a small proportion only of patients with EGFR mutations in NSCLC. This absence of OS benefit even in genotypebased selection of patients raises serious questions and concerns whether the current biologic agents' generation will have the capacity to improve long-term survival and cure rates in the adjuvant setting. ${ }^{4}$ Trastuzumab is providing an OS benefit in metastatic setting for HER2positive breast cancer and gastric cancer and in the adjuvant setting for breast cancer represents an isolated success. However, here there is skepticism because the net

TABLE 1 Examples of efficacy and resistance to targeted agents added to systemic chemotherapy in the treatment of solid cancers

\begin{tabular}{llll}
\hline Agent & Adjuvant setting & Metastatic setting & PFS benefit \\
\cline { 3 - 4 } & Evidence of benefit & OS benefit & \\
\hline Trastuzumab & Breast cancer & $\begin{array}{l}\text { Breast cancer } \\
\text { Gastric cancer }\end{array}$ & Only for wild-type KRAS \\
Cetuximab or panitumumab & NA & No OS benefit in CRC & Only for NSCLC with EGFR mutations \\
Gefitinib or erlotinib & NA & No OS benefit in NSCLC & Modest PFS benefit \\
Bevacizumab & NA & No OS benefit in breast cancer & Withdrawing by FDA* \\
\hline
\end{tabular}

$O S$ overall survival, $P F S$ progression-free survival, $N A$ not available, $C R C$ colorectal cancer, $N S C L C$ non-small cell lung cancer, $F D A$ Food and Drug Administration

* Based on new data from more recent randomized trials FDA has withdrawn bevacizumab in the treatment of metastatic breast cancer 
response rate of trastuzumab is approximately $10 \%$, and longer follow-up is needed to conclude whether this monoclonal antibody can provide cure or has only a recurrence-delaying effect. ${ }^{10}$

\section{Understanding Drug Resistance}

Identifying the causes of treatment failure and highlighting the molecular mechanisms of nonresponsiveness to biologically targeted agents is fundamental for innovation design of the next-generation, more effective biologic agents. A series of reasons explain the limitations of current biologics.

Emerging evidence reveals that the landscape of mutations and deregulated signaling pathways underlying cancer development, progression, and metastasis of solid cancers is much more complicated than we could have imagined. First, recent studies using massively parallel DNA sequencing technology have revealed that not only point mutations such as nucleotide insertions, deletions, and SNPs but also genomic rearrangements and copy number changes are involved in cancer. ${ }^{1-3}$ Although the costs for full-genome sequence may drop to approximately $\$ 1000$ USD in the next few years, allowing thousands of cancer genomes to be completely sequenced, major challenges raise uncertainty. The discrimination between causal (driver) and noncausal (passengers) mutations still remains a challenge. However, the next bigger problem will be to explore and understand the functional role of mutations in the nonprotein coding genome. ${ }^{2,3}$ A second and even greater problem is how to understand the complex genegene, protein-protein, gene-environment, and intratumoral cell-cell interactions in a timely dynamic process. It is thought that the inference of an oncological outcome, namely tumorigenesis or metastatic recurrence is driven by complex molecular networks rather than a simple linear relationship between genetic alterations and phenotype. ${ }^{5,10}$

\section{GENOTYPE-PHENOTYPE MAP}

Both genotype and phenotype complete data are crucial in the effort to predict risk of recurrence and survival. Several studies have already identified a large number of mutations and genes involved in cancer development and metastasis. With less expensive and refined sequencing technology, the number of whole and partial cancer genomes sequence will be dramatically increased, improving the catalog of driver mutations including point mutations rearrangements and copy-number changes. From a phenotype perspective, high-quality clinical, pathological, therapeutic, and follow-up data (phenotype) are available from large-scale RCTs and databases. Although still in its infancy, effort is underway in how to link a phenotypic event (recurrence, death) with genetic alterations (genotype). ${ }^{10}$ Genomic data offer the possible guidance for personalized treatment, and the unique responses of cancer subjects to these same treatments may hint that these similar cancer types hold different genetic markers.

The understanding of a nonlinear complex relationship between genotype and phenotype is still a major problem. However, rapid progress and collaboration between researchers in biomedical and mathematical sciences may overcome current challenges. Several computational strategies are being developed to predict gene-gene and gene-environment interactions. ${ }^{4,5}$ Bionetworks modeling represents one of the most fascinating fields toward a genotype-phenotype-based personalized medicine. ${ }^{9}$ Efforts are underway to integrate genotyping and molecular data into molecular networks modeling to predict outcomes. ${ }^{5,10}$ Systems biology approach shapes a new way to understand complex biological systems such as individual tumor, host, and environment. ${ }^{4}$ The goal is to link genomics data with clinical data for understanding why some patients respond to therapy and are cured whereas others do not respond to therapy, experiencing fatal metastatic recurrence. Given that the mutations catalog has to be completed, bionetworks modeling with emphasis on available completed clinical data might lead more quickly to the development of the next generation of network-based biomarkers and biologically targeted agents. ${ }^{10}$

Beyond the ERBB family EGFR (or HER1), ERBB2 (HER2), ERBB3 (HER3), and VEGF, a crucial role in cancer with a varied nature among patients with the same tumor and stage may also be played by other signaling pathways such as for example Wnt, Notch, Hedgehog, and TGF. ${ }^{10}$ Indeed, a recent study using new sequencing technology has revealed that in pancreatic cancer, approximately 12 signaling pathways are activated per patient and there is a widespread variability of deregulated downstream pathways. Given the interconnections of the signaling pathways components and the deregulated pathway's heterogeneity, it is important not only to characterize which pathways are activated to which patient but also which is the inference of signaling pathway interactions. Therefore, a systems biology approach to understand signaling pathway networks for the prediction of signal transduction that includes input (receptors)-intracellular signaling pathways network-output (nucleus) is crucial for a new generation of biomarkers and biologic agents.

\section{CONCLUSIONS}

Adequate surgery with R0 resection with or without radiotherapy depending on cancer type and stage and 
adjuvant systemic chemotherapy have been standardized, improving overall survival and cure rates of patients with solid cancers in the adjuvant setting. In this setting, targeted therapy has demonstrated an isolated success of trastuzumab when it is added to adjuvant chemotherapy for breast cancer only. In the metastatic setting, modern chemotherapy significantly prolongs overall survival. Yet the addition of targeted agents to chemotherapy in metastatic cancer has mostly improved PFS but not OS. This lack of OS benefit in the metastatic setting raises concerns on the ability of the present generation of targeted agents to improve OS and cure rates in the adjuvant setting.

At the end of the first postgenomic decade with an explosion in whole-genome sequencing technology, genetics and genomics data show at an unprecedented level that solid cancers are much more complex and heterogeneous than we have imagined. This evidence from "big" biology, nearly essentially, shapes innovation strategies toward completion of somatic and heritable driver mutations catalog and understanding the complex genotypephenotype map. Intellectual conceptual designs in how to use next-generation DNA sequencing technology data for a next-generation of robust biomarkers and effective biologic drugs now create a scientifically rational promise.

\section{REFERENCES}

1. The human genome at ten. Nature. 2010;464:649-50.

2. Ledford H. Big science: the cancer genome challenge. Nature. 2010;464:972-4.

3. Check HE. Human genome at ten: life is complicated. Nature. 2010;464:664-7.

4. Roukos DH. Systems medicine: a real approach for future personalized oncology? Pharmacogenomics. 2010;11:283-7.

5. Schadt EE. Molecular networks as sensors and drivers of common human diseases. Nature. 2009;461:218-23.

6. Collins F. Has the revolution arrived? Nature. 2010;464:674-5.

7. Venter JC. Multiple personal genomes await. Nature. 2010;464: 676-7.

8. Lin K, Lipsitz R, Miller T, Janakiraman S; U.S. Preventive Services Task Force. Benefits and harms of prostate-specific antigen screening for prostate cancer: an evidence update for the U.S. Preventive Services Task Force. Ann Intern Med. 2008;149: 192-9.

9. Roukos DH. Bionetworks-based personalized medicine versus comparative-effectiveness research or harmonization of both in cancer management? Expert Rev Mol Diagn. 2010;10:247-50.

10. Roukos DH. Novel clinico-genome network modeling for revolutionizing genotype-phenotype-based personalized cancer care. Expert Rev Mol Diagn. 2010;10:33-48. 